Research Article

\title{
Socio-demographic and Health Profile of Schedule Castes of Patna, Vaishali and Nalanda Bihar, India
}

\author{
Faiyaz Ahmad', Shabana Khatoon', Mumtaz Ahmad', Md Manzar Alam', Hashmat Imam', \\ Shagufta Parveen ${ }^{2}$, Tamanna Nazli', Abdul Raheem ${ }^{2}$, Anirban Goswami', Md Ishtiyaque Alam
}

${ }^{1}$ Regional Research, Institute of Unani Medicine, Patna, Bihar, India.

${ }^{2}$ Central Council for Research in Unani Medicine (CCRUM), New Delhi, India.

DOI: https://doi.org/10.24321/2454.325X.202102

\section{I $\quad \mathbf{N} \quad \mathbf{F} \quad \mathbf{O}$}

\section{Corresponding Author:}

Faiyaz Ahmad, RRIUM, Patna, Bihar, India.

E-mail Id:

faizienadira@gmail.com

Orcid Id:

https://orcid.org/0000-0002-6192-5491

How to cite this article:

Ahmad F, Khatoon S, Ahmad M, Alam MM, Imam

H, Parveen S, Nazli T, Raheem A, Goswami A, Alam MI. Socio-demographic and Health Profile of Schedule Castes of Patna, Vaishali and Nalanda Bihar, India. Int J Preven Curat Comm Med. 2021; 7(1): 11-19.

Date of Submission: 2021-03-01

Date of Acceptance: 2021-03-27

\section{$\begin{array}{llllllll}\mathbf{A} & \mathbf{B} & \mathbf{S} & \mathbf{T} & \mathbf{R} & \mathbf{A} & \mathbf{C} & \mathbf{T}\end{array}$}

Background: Patna and peripheral districts (Nalanda and Vaishali) have some dominated area of SC and ST population but the distribution and demographic details of these areas are not properly documented. The main objective of the paper is to analyze the status of the scheduled castes of Patna, with respect to size of the population, sex ratio, literacy level, marital status, occupation and income.

Methodology: A pre-tested questionnaire containing information about demographic particulars like caste, age, gender, educational qualification, occupation and monthly income was administered through Mobile health care OPDs under the SCSP. Survey was conducted between the years 2018-2020. These data are shown to be suitable for evaluating the impact of demographic and socio-economic elements on regular health examinations.

Result: The analysis of data clearly indicates that there were significant differences with the age group 13-20, 21-30 and $>60$, caste, with religion of Hindu and Muslim, educational status regarding semi-literate and graduate or above, Mizaj, diet habit and there were no significant differences with respect to gender, religion and addiction behaviour are noticed. By presenting the methods used in this survey and by describing the enquiries mentioned in the dataset, this article aims to promote data-collecting methodologies that can help policy-makers and health communicators derive practical conclusions.

Conclusion: It can be concluded that there is significant difference in the age group of SC population. While there were no significant differences with respect to gender, religion and addiction behaviour was noticed. Most of the SC population belongs to balghami temperament and most preferred food habit of them is mixed mostly.

Keywords: Patna, Survey, Scheduled caste Sub Plan, Unani Medicine, AYUSH 


\section{Introduction}

The scheduled caste (SC) population constituted 16.2 per cent of the total population in Census 2001 and increased marginally to around 16.6 per cent in Census 2011. People belonging to $\mathrm{SC}$ communities, by and large, are spread all over the country, with about 80 per cent of them living in rural areas. Around half of the SC population is concentrated in five States - Uttar Pradesh, West Bengal, Tamil Nadu, Andhra Pradesh and Bihar. ${ }^{1}$

During the period of British rule in the Indian subcontinent, they were known as the oppressed class. After independence, the Constituent Assembly continued the prevailing definition of Scheduled Castes and Tribes, giving (via articles 341 and 342 ) the president of India and governors of the states passed a mandate to compile a full listing of castes and tribes. ${ }^{2,3}$ SC people also known as Dalits are socially excluded in India and face discrimination on the basis of their position at the very bottom of the Indian caste system.

The Scheduled Castes are those communities that are scheduled in accordance with clause (1) of article 341 of the Constitution. ${ }^{4}$ The schedule castes are comparatively backward in occupational, educational, social, cultural, religious and political sector. ${ }^{5}$ According to census of 2011 the scheduled caste population of India is $16.63 \%$ (201378372) of total Indian population (1210854977) out of which $51.41 \%$ are male while $48.59 \%$ are female. The scheduled caste population in Bihar is $15.91 \%(1,65,67,325)$ of total Population of Bihar $(10,40,99,452)$, out of which $51.95 \%$ are male while $48.05 \%$ are female. ${ }^{6}$ The scheduled caste population of Patna district is $15.77 \%$ (920918) of total population (5838465) of Patna district. ${ }^{7}$ As far as distribution on SC in districts of Patna is taken under consideration, it is $21.11 \%(738,031)$ of total population $(3,495,021)$ in Vaishali district, out of which $51.95 \%$ are male while $48.05 \%$ are female ${ }^{8}$ in Nalanda district, SC population is $21.11 \%$ $(607,672)$ of total population i.e., $2,877,653 .{ }^{9}$

The strategy of Scheduled Caste-Sub plan (SC-SP) envisages to channelize the flow of outlays and benefits from all the sectors of development in the annual plans of states/UTs and Central Ministries at least in proportion both in physical and financial terms.

The Central Council for Research in Unani Medicine (CCRUM) is an apex autonomous research organization functioning under the ministry of AYUSH, Government of India. The council has been directed to implement a health programme under Scheduled Caste-Sub plan (SCSP) for the benefit of SC population. ${ }^{10}$ The objectives of the program are to screen/ examine the SC population for their health status in the OPD as well as in the health camps and to provide Unani treatment to the patients suffering from different diseases. Also, it aims to create awareness among the masses on preventive, promotive and curative health aspects through lectures, group meetings, health camps and distribution of literature among SC population. In this connection, the Regional Research Institute of Unani Medicine Patna, a peripheral institute of CCRUM organised Mobile Healthcare Programme in SC dominated areas of state.

Present study is a compilation of demographic details collected in the Mobile Healthcare Program under SCSP in Patna.

\section{Objective}

The objective of the study is to enumerate and analyse the demographic characteristics of scheduled caste populated area of ten different selected spots at Patna, Vaishali and Nalanda districts of Bihar state.

\section{Methodology}

A mobile health care team from Regional Research Institute of Unani Medicine, Patna conducted weekly visit at ten selected spots in scheduled caste populated area of Patna, Vaishali and Nalanda districts of Bihar during year 2018-19 to 2019-20 where OPD was being conducted and screening as well as treatment of patients were done and data was collected. Also, a questionnaire containing demographic information like caste, age, gender, educational qualification, occupation and monthly income was administered by paying house to house visits. After collection of data, it was double entered in case record form (CRF) \& Microsoft Excel Sheet. A clean database was generated and analysed using R-4.0.0., data was presented by using frequency tables to find out the association between categorical variables Chi-square test was used. P-value less than 0.05 were considered as statistically significant.

\section{Results}

A total number of 11,621 participants were taken under the Mobile Health care Programme under SCSP at Patna in various $\mathrm{SC}$ dominated spots namely Alampur, Alawalpur, Barni, Dhobghatti, Diawan, Gurichak, Keora, Lakhna, P. Bigha and Phetiya. Majority of 1586 participants were from spot Alampur, 538 from Alawalpur, 2334 from Barni, 559 from Dhobghatti, 449 from Diawan, 1981 from Gaurichak, 482 from Keora, 1648 from Lakhna and 1566 from Phetiya. Minimum number of participants belong to spot namely P. Bigha (Table 1).

Among 11,621 respondents in participating 10 spots, mean age of participants at Alampur was $39.64 \pm 20.47$, while in Alwalpur, Barni, Dhobghatti, Diawan, Gurichak, Keora, Lakhna, P. Bigha and Phetiya, mean age of the participants were $49.58 \pm 18.22,47.9 \pm 19.12,48.42 \pm 17.44,45.6 \pm$ $17.4,46.77 \pm 19.28,52.35 \pm 15.38,46.29 \pm 18.57,49.94$ $\pm 18.3,42.81 \pm 19.54$ and $45.95 \pm 19.24$ respectively. Majority of participants 255 (16.08\%) and 173 (32.16\%) 
were above 60 years at Alampur and Alwalpur respectively. $228(14.38 \%)$ participants are of age group $13-20$ years at Alampur, 104(19.33\%) and $147(30.75 \%)$ participants come under age group of 51-60 years at Alwalpur and P. Bigha respectively. There were significant differences throughout the spots with the age group 13-20, 21-30 and $>60(x 2$ $=17.64, p$-value $<0.001)$, ( $\chi 2=6$, $p$-value <0.001) and $(\chi 2$ =24.04, p-value<0.001) (Table 2).

Out of total respondents, 5156 (44.37\%) were males and 6465 (55.63\%) were females; the large majority of male $853(54.47 \%)$ were found in Phetiya and the large majority of female 1461 (62.6\%) were found in Barni. There was no significant difference throughout the spots with gender $(\chi 2$ =0.9, p-value $=0.34)$ (Table 3).

Among 11621 respondents, total scheduled caste population were 10300 (88.63\%), there was no any ST caste found and other castes were 1321 (11.37\%); majority of scheduled caste were found at Pandey Bigha 467 (97.7\%); large majority of other caste were found at Lakhna 270 (16.38\%). There was significant difference throughout the spots with caste ( $\chi 2=18.78$, p-value <0.001) (Table 4).

Total numbers of unmarried were 1758 (15.13\%) and married were 9863 (84.87\%). Majority of unmarried participants were found at Alampur 395 (24.91\%), while majority of married were found at Keora 470 (97.51\%). There was significant difference throughout the spots with marital status ( $\chi 2=14.95$, $p$-value $<0.001$ ). No divorced and separated couple was observed among the participating population (Table 5).

Of the 11621 respondents surveyed majority were following Hinduism (94.72\%) and only few were muslim (5.25\%). Majority of Hindu population were found at Alawalpur 538 (100\%), while maximum of Muslims were found at Alampur 190 (11.98\%). There were significant difference in the spots with context to religion Hinduism and Islam ( $\chi 2=31.47$ and 31.25 , p-value $<0.001$ and $<0.001$ respectively) but there was no significant difference throughout the spots with others religion ( $\chi 2=0.19, p$-value $=0.66$ ) (Table 6).

Educational status was assessed by categorizing several grades as illiterate, semi-literate, primary schooling, high schooling, intermediate and graduate. It has been observed that 5927 (51\%) participants were illiterate followed by 3217 (27.68\%) semi-literate. 1125 (9.68\%) participants were having education of primary school, while 688 (5.92\%) participants were high school and $436(3.75 \%)$ intermediate. Only 228 (1.96\%) participants were graduated or having higher degree while illiterates were mainly belonged to Pandey Bigha 352 (73.64\%), semi-literates were found at
Lakhna 568 (34.47\%). Most of the participants 313 (19.74\%) at Alampur were educated to primary school and 37 (8.24\%) participants from Diawan were educated upto High school. Among graduates, most of the participants were from Gaurichak 69 (3.48\%). There was significant difference in the spots with Educational status regarding especially in variable semi-literate and graduate or above $(\chi 2=5.64$, p-value <002) and ( $\chi 2=4.21, p$-value <0.04) (Table 7).

Among 11621 respondents in all adopted spots, maximum number of participants 7252 (62.4\%) were of Balghami mizaj 1609 (13.85\%) were of Damvi (sanguine) temperament. A total of 1418 (12.2\%) were of safravi mizaj and 1342 (11.55\%) were saudavi. Majority of Balghami temperament participants were from Pandey Bigha 439 (91.84\%) while most of the Damvi temperament participants were from Alampur. There is significant difference assessed in mizaj of the participants in adopted spots (Table 8).

Most of the participants 10484 (90.22\%) preferred Non vegetarian/ Mixed diet, followed by vegetarian who were 1039 (8.94\%), while rest of the participants $98(0.84 \%)$ were vegetarian along with positive consumption of egg. Majority of non-vegetarian group of participants 481 (99.79\%) were from Keora and Pandey Bigha. There was significant difference in diet habit of participants, resident of ten adopted spots. ( $\chi 2=18.9, p$-value $<0.001)$ and $(\chi 2$ $=20.73$, p-value <0.001) (Table 9).

Majority of the participants 5486 (47.21\%) were housewives, this may be due to a greater number of female participants. Among the males, most of the participants 1988 (17.11\%) were Agricultural Labourer followed by 1245 (10.71\%) participants who were not engaged in any occupation, 771 (6.63\%) were Unskilled Labourer while skilled labourer were mere $1.45 \%$. There were 1578 (13.58\%) students among participants while $0.28 \%$ were retired from their jobs. Majority of agricultural labourer 112 (23.24\%) belongs to Keora, while most of the students were resident of Alampur. There is significant difference observed at baseline while assessing the occupational status of the participants of 10 adopted spots ( $\chi 2=24.13, p$-value $<0.001),(\chi 2=9.09$, $\mathrm{p}$-value $<0.001),(\chi 2=5.41, \mathrm{p}$-value $<0.02)$ and $(\chi 2=5.3$, p-value $<0.020$ ) respectively (Table 10 ).

Among 11,621 participants, majority of the population 8086 $(69.58 \%)$ were not having history of any addiction. Those who have habit of addiction, 3451 (29.7\%) were mainly tobacco chewer, while very few among them reported addiction to bhang, snuff, alcohol and smoking etc. most of the non-addictive population among the participants were resident of Barni. There was no significant difference throughout the spots with addiction behaviour. 
Ahmad F et al.

Table I.Age distribution of Participants in different Adopted Spots

\begin{tabular}{|c|c|c|c|c|c|c|c|c|c|c|c|c|c|}
\hline \multirow{2}{*}{ Variable } & \multicolumn{10}{|c|}{ Name of the Spot } & \multirow{2}{*}{$\begin{array}{c}\text { Total } \\
\mathbf{N}\end{array}$} & \multirow{3}{*}{$\mathrm{X} 2$} & \multirow{3}{*}{$p$-value } \\
\hline & Alampur & Alawalpur & Barni & Dhobghatti & Diawan & Gaurichak & Keora & Lakhna & P. Bigha & Phetiya & & & \\
\hline Age in years & $n=1586$ & $n=538$ & $n=2334$ & $n=559$ & $n=449$ & $n=1981$ & $n=482$ & $n=1648$ & $n=478$ & $n=1566$ & $N=11621$ & & \\
\hline $1-12$ & $\begin{array}{c}165 \\
(10.4 \%)\end{array}$ & 30 (5.58\%) & $\begin{array}{c}133 \\
(5.7 \%)\end{array}$ & $12(2.15 \%)$ & $9(2 \%)$ & $107(5.4 \%)$ & $4(0.83 \%)$ & $\begin{array}{c}86 \\
(5.22 \%)\end{array}$ & $9(1.88 \%)$ & 85 (5.43\%) & $640(5.51 \%)$ & 0.01 & 0.92 \\
\hline $13-20$ & $\begin{array}{c}228 \\
(14.38 \%)\end{array}$ & 19 (3.53\%) & $\begin{array}{c}149 \\
(6.38 \%)\end{array}$ & $38(6.8 \%)$ & $\begin{array}{c}40 \\
(8.91 \%)\end{array}$ & $\begin{array}{c}152 \\
(7.67 \%)\end{array}$ & 9 (1.87\%) & $\begin{array}{c}122 \\
(7.4 \%)\end{array}$ & 33 (6.9\%) & $\begin{array}{c}192 \\
(12.26 \%)\end{array}$ & $982(8.45 \%)$ & 17.64 & $0.001 *$ \\
\hline $21-30$ & $\begin{array}{c}242 \\
(15.26 \%)\end{array}$ & 47 (8.74\%) & $\begin{array}{c}247 \\
(10.58 \%)\end{array}$ & $66(11.81 \%)$ & $\begin{array}{c}60 \\
(13.36 \%)\end{array}$ & $\begin{array}{c}225 \\
(11.36 \%)\end{array}$ & $\begin{array}{c}47 \\
(9.75 \%)\end{array}$ & $\begin{array}{c}193 \\
(11.71 \%)\end{array}$ & $54(11.3 \%)$ & $\begin{array}{c}225 \\
(14.37 \%)\end{array}$ & $\begin{array}{c}1406 \\
(12.1 \%)\end{array}$ & 6 & 0.01 \\
\hline $31-40$ & $\begin{array}{c}232 \\
(14.63 \%)\end{array}$ & $\begin{array}{c}84 \\
(15.61 \%)\end{array}$ & $\begin{array}{c}323 \\
(13.84 \%)\end{array}$ & $93(16.64 \%)$ & $\begin{array}{c}87 \\
(19.38 \%)\end{array}$ & $\begin{array}{c}272 \\
(13.73 \%)\end{array}$ & $\begin{array}{c}87 \\
(18.05 \%)\end{array}$ & $\begin{array}{c}234 \\
(14.2 \%)\end{array}$ & $\begin{array}{c}79 \\
(16.53 \%)\end{array}$ & $\begin{array}{c}273 \\
(17.43 \%)\end{array}$ & $\begin{array}{c}1764 \\
(15.18 \%)\end{array}$ & 0.08 & 0.78 \\
\hline $41-50$ & $\begin{array}{c}232 \\
(14.63 \%)\end{array}$ & $\begin{array}{c}81 \\
(15.06 \%)\end{array}$ & $\begin{array}{c}332 \\
(14.22 \%)\end{array}$ & 83 (14.85\%) & $\begin{array}{c}77 \\
(17.15 \%)\end{array}$ & $\begin{array}{c}327 \\
(16.51 \%)\end{array}$ & $\begin{array}{c}70 \\
(14.52 \%)\end{array}$ & $\begin{array}{c}278 \\
(16.87 \%)\end{array}$ & $\begin{array}{c}57 \\
(11.92 \%)\end{array}$ & $\begin{array}{c}264 \\
(16.86 \%)\end{array}$ & $\begin{array}{c}1801 \\
(15.5 \%)\end{array}$ & 0.08 & 0.78 \\
\hline $51-60$ & $\begin{array}{c}232 \\
(14.63 \%)\end{array}$ & $\begin{array}{c}104 \\
(19.33 \%)\end{array}$ & $\begin{array}{c}533 \\
(22.84 \%)\end{array}$ & $\begin{array}{c}132 \\
(23.61 \%)\end{array}$ & $\begin{array}{c}95 \\
(21.16 \%)\end{array}$ & $\begin{array}{c}390 \\
(19.69 \%)\end{array}$ & $\begin{array}{c}131 \\
(27.18 \%)\end{array}$ & $\begin{array}{c}350 \\
(21.24 \%)\end{array}$ & $\begin{array}{c}99 \\
(20.71 \%)\end{array}$ & $\begin{array}{c}241 \\
(15.39 \%)\end{array}$ & $\begin{array}{c}2307 \\
(19.85 \%)\end{array}$ & 0.1 & 0.75 \\
\hline$>60$ & $\begin{array}{c}255 \\
(16.08 \%)\end{array}$ & $\begin{array}{c}173 \\
(32.16 \%)\end{array}$ & $\begin{array}{c}617 \\
(26.44 \%)\end{array}$ & $\begin{array}{c}135 \\
(24.15 \%)\end{array}$ & $\begin{array}{c}81 \\
(18.04 \%)\end{array}$ & $\begin{array}{c}508 \\
(25.64 \%)\end{array}$ & $\begin{array}{c}134 \\
(27.8 \%) \\
\end{array}$ & $\begin{array}{c}385 \\
(23.36 \%)\end{array}$ & $\begin{array}{c}147 \\
(30.75 \%)\end{array}$ & $\begin{array}{c}286 \\
(18.26 \%)\end{array}$ & $\begin{array}{c}2721 \\
(23.41 \%)\end{array}$ & 24.04 & $0.001 *$ \\
\hline Mean \pm SD & $\begin{array}{c}39.64 \\
\pm 20.47\end{array}$ & $\begin{array}{c}49.58 \\
\pm 18.22\end{array}$ & $\begin{array}{l}47.9 \pm \\
19.12\end{array}$ & $\begin{array}{c}48.42 \pm \\
17.44\end{array}$ & $\begin{array}{c}45.6 \pm \\
17.4\end{array}$ & $\begin{array}{c}46.77 \pm \\
19.28\end{array}$ & $\begin{array}{c}52.35 \pm \\
15.38\end{array}$ & $\begin{array}{c}46.29 \pm \\
18.57\end{array}$ & $49.94 \pm 18.3$ & $\begin{array}{c}42.81 \pm \\
19.54\end{array}$ & $\begin{array}{c}45.95 \pm \\
19.24\end{array}$ & - & - \\
\hline
\end{tabular}

Note: $X^{2}=$ Chi-square, $n=$ number of respondents, ${ }^{*} p$ value $<0.05=$ statistically significant.

Table 2.Gender wise distribution of Participants in different Adopted Spots

\begin{tabular}{|c|c|c|c|c|c|c|c|c|c|c|c|c|c|}
\hline \multirow{2}{*}{ Variable } & \multicolumn{10}{|c|}{ Name of the Spots } & \multirow{2}{*}{$\begin{array}{c}\text { Total } \\
\mathbf{N}\end{array}$} & \multirow{3}{*}{$\mathrm{X} 2$} & \multirow{3}{*}{ p-value } \\
\hline & Alampur & Alawalpur & Barni & Dhobghatti & Diawan & Gaurichak & Keora & Lakhna & P. Bigha & Phetiya & & & \\
\hline Gender & $n=1586$ & $n=538$ & $n=2334$ & $n=559$ & $n=449$ & $n=1981$ & $n=482$ & $n=1648$ & $n=478$ & $n=1566$ & $\mathrm{~N}=11621$ & & \\
\hline Male & $\begin{array}{c}822 \\
(51.83 \%)\end{array}$ & $\begin{array}{c}228 \\
(42.38 \%)\end{array}$ & $\begin{array}{c}873 \\
(37.4 \%)\end{array}$ & $\begin{array}{c}244 \\
(43.65 \%)\end{array}$ & $\begin{array}{c}169 \\
(37.64 \%)\end{array}$ & $\begin{array}{c}785 \\
(39.63 \%)\end{array}$ & $\begin{array}{c}188 \\
(39 \%)\end{array}$ & $\begin{array}{c}812 \\
(49.27 \%)\end{array}$ & $\begin{array}{c}182 \\
(38.08 \%)\end{array}$ & $\begin{array}{c}853 \\
(54.47 \%)\end{array}$ & $\begin{array}{c}5156 \\
(44.37 \%)\end{array}$ & 0.9 & 0.34 \\
\hline Female & $\begin{array}{c}764 \\
(48.17 \%)\end{array}$ & $\begin{array}{c}310 \\
(57.62 \%)\end{array}$ & $\begin{array}{c}1461 \\
(62.6 \%)\end{array}$ & $\begin{array}{c}315 \\
(56.35 \%)\end{array}$ & $\begin{array}{c}280 \\
(62.36 \%)\end{array}$ & $\begin{array}{c}1196 \\
(60.37 \%)\end{array}$ & $\begin{array}{c}294 \\
(61 \%)\end{array}$ & $\begin{array}{c}836 \\
(50.73 \%)\end{array}$ & $\begin{array}{c}296 \\
(61.92 \%)\end{array}$ & $\begin{array}{c}713 \\
(45.53 \%)\end{array}$ & $\begin{array}{c}6465 \\
(55.63 \%)\end{array}$ & & \\
\hline
\end{tabular}

Note: $\mathrm{X}^{2}=$ Chi-square, $\mathrm{n}=$ number of respondents, ${ }^{*} \mathrm{p}$ value $<0.05$ = statistically significant.

ISSN: 2454-325X

DOI: https://doi.org/10.24321/2454.325X.202102 
Table 3.Caste wise distribution of Participants in different Adopted Spots

\begin{tabular}{|c|c|c|c|c|c|c|c|c|c|c|c|c|c|}
\hline \multirow{2}{*}{ Variable } & \multicolumn{10}{|c|}{ Name of the Spots } & \multirow{2}{*}{$\begin{array}{l}\text { Total } \\
\text { n (\%) }\end{array}$} & \multirow{3}{*}{$\mathbf{X 2}$} & \multirow{3}{*}{ p-value } \\
\hline & Alampur & Alawalpur & Barni & Dhobghatti & Diawan & Gaurichak & Keora & Lakhna & P. Bigha & Phetiya & & & \\
\hline Caste & $n=1586$ & $n=538$ & $n=2334$ & $n=559$ & $n=449$ & $n=1981$ & $n=482$ & $n=1648$ & $n=478$ & $n=1566$ & $\mathrm{~N}=11621$ & & \\
\hline $\mathrm{SC}$ & $\begin{array}{c}1352 \\
(85.25 \%)\end{array}$ & $\begin{array}{c}508 \\
(94.42 \%) \\
\end{array}$ & $\begin{array}{c}2112 \\
(90.49 \%)\end{array}$ & $\begin{array}{c}535 \\
(95.71 \%)\end{array}$ & $\begin{array}{c}417 \\
(92.87 \%) \\
\end{array}$ & $\begin{array}{c}1722 \\
(86.93 \%)\end{array}$ & $\begin{array}{c}456 \\
(94.61 \%) \\
\end{array}$ & $\begin{array}{c}1378 \\
(83.62 \%)\end{array}$ & $\begin{array}{c}467 \\
(97.7 \%)\end{array}$ & $\begin{array}{c}1353 \\
(86.4 \%)\end{array}$ & $\begin{array}{c}10300 \\
(88.63 \%)\end{array}$ & \multirow{3}{*}{18.78} & \multirow{3}{*}{$0.001^{*}$} \\
\hline ST & $0(0 \%)$ & $0(0 \%)$ & $0(0 \%)$ & $0(0 \%)$ & $0(0 \%)$ & $0(0 \%)$ & $0(0 \%)$ & $0(0 \%)$ & $0(0 \%)$ & $0(0 \%)$ & $0(0 \%)$ & & \\
\hline Other & $\begin{array}{c}234 \\
(14.75 \%)\end{array}$ & $30(5.58 \%)$ & $\begin{array}{c}222 \\
(9.51 \%)\end{array}$ & 24 (4.29\%) & $32(7.13 \%)$ & $\begin{array}{c}259 \\
(13.07 \%)\end{array}$ & $26(5.39 \%)$ & $\begin{array}{c}270 \\
(16.38 \%)\end{array}$ & $\begin{array}{c}11 \\
(2.3 \%)\end{array}$ & $\begin{array}{c}213 \\
(13.6 \%)\end{array}$ & $\begin{array}{c}1321 \\
(11.37 \%)\end{array}$ & & \\
\hline
\end{tabular}

Note: $\mathrm{X}^{2}=$ Chi-square, $\mathrm{n}=$ number of respondents, ${ }^{*} \mathrm{p}$ value $<0.05=$ statistically significant.

Table 4.Distribution of Participants according to Marital Status in different Adopted Spots

\begin{tabular}{|c|c|c|c|c|c|c|c|c|c|c|c|c|c|}
\hline \multirow{2}{*}{ Variable } & \multicolumn{10}{|c|}{ Name of the Spots } & & \multirow{3}{*}{$\mathbf{X} 2$} & \multirow{3}{*}{ p-value } \\
\hline & Alampur & Alawalpur & Barni & Dhobghatti & Diawan & Gaurichak & Keora & Lakhna & P. Bigha & Phetiya & Total & & \\
\hline $\begin{array}{c}\text { Marital } \\
\text { status }\end{array}$ & $n=1586$ & $n=538$ & $n=2334$ & $n=559$ & $n=449$ & $n=1981$ & $n=482$ & $n=1648$ & $n=478$ & $n=1566$ & $n=11621$ & & \\
\hline Unmarried & $\begin{array}{c}395 \\
(24.91 \%)\end{array}$ & $50(9.29 \%)$ & $\begin{array}{c}300 \\
(12.85 \%)\end{array}$ & $55(9.84 \%)$ & $\begin{array}{c}50 \\
(11.14 \%)\end{array}$ & $\begin{array}{c}295 \\
(14.89 \%)\end{array}$ & $\begin{array}{c}12 \\
(2.49 \%) \\
\end{array}$ & $\begin{array}{c}225 \\
(13.65 \%)\end{array}$ & $\begin{array}{c}50 \\
(10.46 \%)\end{array}$ & $\begin{array}{c}326 \\
(20.82 \%)\end{array}$ & $\begin{array}{c}1758 \\
(15.13 \%)\end{array}$ & $\begin{array}{c}14.95 \\
-\end{array}$ & $\begin{array}{c}0.001 * \\
-\end{array}$ \\
\hline Married & $\begin{array}{c}1191 \\
(75.09 \%)\end{array}$ & $\begin{array}{c}488 \\
(90.71 \%) \\
\end{array}$ & $\begin{array}{c}2034 \\
(87.15 \%) \\
\end{array}$ & $\begin{array}{c}504 \\
(90.16 \%) \\
\end{array}$ & $\begin{array}{c}399 \\
(88.86 \%)\end{array}$ & $\begin{array}{c}1686 \\
(85.11 \%) \\
\end{array}$ & $\begin{array}{c}470 \\
(97.51 \%) \\
\end{array}$ & $\begin{array}{c}1423 \\
(86.35 \%)\end{array}$ & $\begin{array}{c}428 \\
(89.54 \%)\end{array}$ & $\begin{array}{c}1240 \\
(79.18 \%)\end{array}$ & $\begin{array}{c}9863 \\
(84.87 \%)\end{array}$ & & \\
\hline Divorced & $0(0 \%)$ & $0(0 \%)$ & $0(0 \%)$ & $0(0 \%)$ & $0(0 \%)$ & $0(0 \%)$ & $0(0 \%)$ & $0(0 \%)$ & $0(0 \%)$ & $0(0 \%)$ & $0(0 \%)$ & & \\
\hline $\begin{array}{l}\text { Widow/ } \\
\text { Widower }\end{array}$ & $0(0 \%)$ & $0(0 \%)$ & $0(0 \%)$ & $0(0 \%)$ & $0(0 \%)$ & $0(0 \%)$ & $0(0 \%)$ & $0(0 \%)$ & $0(0 \%)$ & $0(0 \%)$ & $0(0 \%)$ & - & - \\
\hline Separated & $0(0 \%)$ & $0(0 \%)$ & $0(0 \%)$ & $0(0 \%)$ & $0(0 \%)$ & $0(0 \%)$ & $0(0 \%)$ & $0(0 \%)$ & $0(0 \%)$ & $0(0 \%)$ & $0(0 \%)$ & - & - \\
\hline
\end{tabular}

Note: $\mathrm{X}^{2}=$ Chi-square, $\mathrm{n}=$ number of respondents, ${ }^{*} \mathrm{p}$ value $<0.05=$ statistically significant. 
Ahmad F et al.

Table 5.Distribution of Participants according to Religion in different Adopted Spots

\begin{tabular}{|c|c|c|c|c|c|c|c|c|c|c|c|c|c|}
\hline \multirow{2}{*}{ Variable } & \multicolumn{10}{|c|}{ Name of the Spots } & \multirow{2}{*}{$\begin{array}{l}\text { Total } \\
\text { n (\%) }\end{array}$} & \multirow{3}{*}{$\mathbf{X 2}$} & \multirow{3}{*}{ p-value } \\
\hline & Alampur & Alawalpur & Barni & Dhobghatti & Diawan & Gaurichak & Keora & Lakhna & P. Bigha & Phetiya & & & \\
\hline Religion & $n=1586$ & $n=538$ & $n=2334$ & $n=559$ & $n=449$ & $n=1981$ & $n=482$ & $n=1648$ & $n=478$ & $n=1566$ & $N=11621$ & & \\
\hline Hinduism & $\begin{array}{c}1396 \\
(88.02 \%)\end{array}$ & $538(100 \%)$ & $\begin{array}{c}2217 \\
(94.99 \%)\end{array}$ & $\begin{array}{c}558 \\
(99.82 \%)\end{array}$ & $\begin{array}{c}419 \\
(93.32 \%)\end{array}$ & $\begin{array}{c}1978 \\
(99.85 \%)\end{array}$ & $\begin{array}{c}461 \\
(95.64 \%)\end{array}$ & $\begin{array}{c}1533 \\
(93.02 \%)\end{array}$ & $\begin{array}{c}472 \\
(98.74 \%)\end{array}$ & $\begin{array}{c}1435 \\
(91.63 \%)\end{array}$ & $\begin{array}{c}11007 \\
(94.72 \%)\end{array}$ & 31.47 & $0.001 *$ \\
\hline Islam & $\begin{array}{c}190 \\
(11.98 \%)\end{array}$ & $0(0 \%)$ & $\begin{array}{c}117 \\
(5.01 \%)\end{array}$ & $1(0.18 \%)$ & $\begin{array}{c}30 \\
(6.68 \%)\end{array}$ & $3(0.15 \%)$ & $\begin{array}{c}21 \\
(4.36 \%)\end{array}$ & $\begin{array}{c}115 \\
(6.98 \%)\end{array}$ & $6(1.26 \%)$ & $\begin{array}{c}127 \\
(8.11 \%)\end{array}$ & $610(5.25 \%)$ & 31.25 & - \\
\hline Sikh & $0(0 \%)$ & $0(0 \%)$ & $0(0 \%)$ & $0(0 \%)$ & $0(0 \%)$ & $0(0 \%)$ & $0(0 \%)$ & $0(0 \%)$ & $0(0 \%)$ & $0(0 \%)$ & $0(0 \%)$ & - & \\
\hline Christian & $0(0 \%)$ & $0(0 \%)$ & $0(0 \%)$ & $0(0 \%)$ & $0(0 \%)$ & $0(0 \%)$ & $0(0 \%)$ & $0(0 \%)$ & $0(0 \%)$ & $0(0 \%)$ & $0(0 \%)$ & - & - \\
\hline
\end{tabular}

Note: $X^{2}=$ Chi-square, $n=$ number of respondents, ${ }^{*} p$ value $<0.05=$ statistically significant.

Table 6.Distribution of Participants according to Educational Status in different Adopted Spots

\begin{tabular}{|c|c|c|c|c|c|c|c|c|c|c|c|c|c|}
\hline \multirow{2}{*}{ Variable } & \multicolumn{10}{|c|}{ Name of the Spots } & Total & \multirow{3}{*}{$\times 2$} & \multirow{3}{*}{ p-value } \\
\hline & Alampur & Alawalpur & Barni & Dhobghatti & Diawan & Gaurichak & Keora & Lakhna & P. Bigha & Phetiya & n (\%) & & \\
\hline $\begin{array}{l}\text { Education } \\
\text { status }\end{array}$ & $n=1586$ & $n=538$ & $n=2334$ & $n=559$ & $n=449$ & $n=1981$ & $n=482$ & $n=1648$ & $n=478$ & $n=1566$ & $n=11621$ & & \\
\hline Illiterate & $\begin{array}{c}841 \\
(53.03 \%)\end{array}$ & $\begin{array}{c}253 \\
(47.03 \%)\end{array}$ & $\begin{array}{c}1135 \\
(48.63 \%)\end{array}$ & $\begin{array}{c}287 \\
(51.34 \%)\end{array}$ & $\begin{array}{c}265 \\
(59.02 \%)\end{array}$ & $\begin{array}{c}1011 \\
(51.03 \%)\end{array}$ & $\begin{array}{c}331 \\
(68.67 \%)\end{array}$ & $\begin{array}{c}764 \\
(46.36 \%)\end{array}$ & $\begin{array}{c}352 \\
(73.64 \%)\end{array}$ & $\begin{array}{c}688 \\
(43.93 \%)\end{array}$ & 5927 (51\%) & 3.57 & 0.06 \\
\hline Semi-literate & $\begin{array}{c}243 \\
(15.32 \%) \\
\end{array}$ & $\begin{array}{c}173 \\
(32.16 \%) \\
\end{array}$ & $\begin{array}{c}775 \\
(33.2 \%) \\
\end{array}$ & $\begin{array}{c}182 \\
(32.56 \%)\end{array}$ & 88 (19.6\%) & $\begin{array}{c}521 \\
(26.3 \%) \\
\end{array}$ & $\begin{array}{c}124 \\
(25.73 \%) \\
\end{array}$ & $\begin{array}{c}568 \\
(34.47 \%) \\
\end{array}$ & $\begin{array}{c}74 \\
(15.48 \%) \\
\end{array}$ & $\begin{array}{c}469 \\
(29.95 \%)\end{array}$ & $\begin{array}{c}3217 \\
(27.68 \%)\end{array}$ & 5.64 & 0.02 \\
\hline $\begin{array}{c}\text { Primary } \\
\text { School }\end{array}$ & $\begin{array}{c}313 \\
(19.74 \%)\end{array}$ & $40(7.43 \%)$ & $\begin{array}{c}193 \\
(8.27 \%) \\
\end{array}$ & $20(3.58 \%)$ & $\begin{array}{c}46 \\
(10.24 \%)\end{array}$ & $\begin{array}{c}178 \\
(8.99 \%)\end{array}$ & $6(1.24 \%)$ & $\begin{array}{c}108 \\
(6.55 \%)\end{array}$ & $23(4.81 \%)$ & $\begin{array}{c}198 \\
(12.64 \%)\end{array}$ & $\begin{array}{c}1125 \\
(9.68 \%) \\
\end{array}$ & 3.25 & 0.07 \\
\hline High School & $\begin{array}{c}104 \\
(6.56 \%)\end{array}$ & $34(6.32 \%)$ & $\begin{array}{c}109 \\
(4.67 \%)\end{array}$ & $32(5.72 \%)$ & 37 (8.24\%) & $\begin{array}{c}116 \\
(5.86 \%)\end{array}$ & $13(2.7 \%)$ & $\begin{array}{c}126 \\
(7.65 \%)\end{array}$ & $14(2.93 \%)$ & $\begin{array}{c}103 \\
(6.58 \%)\end{array}$ & $\begin{array}{c}688 \\
(5.92 \%)\end{array}$ & 0.16 & 0.69 \\
\hline Intermediate & $\begin{array}{c}51 \\
(3.22 \%)\end{array}$ & $21(3.9 \%)$ & $\begin{array}{c}90 \\
(3.86 \%)\end{array}$ & $24(4.29 \%)$ & $6(1.34 \%)$ & $86(4.34 \%)$ & $4(0.83 \%)$ & 55 (3.34\%) & $9(1.88 \%)$ & $90(5.75 \%)$ & $\begin{array}{c}436 \\
(3.75 \%)\end{array}$ & 0.04 & 0.84 \\
\hline $\begin{array}{c}\text { Graduate or } \\
\text { above }\end{array}$ & $\begin{array}{c}34 \\
(2.14 \%)\end{array}$ & $17(3.16 \%)$ & $\begin{array}{c}32 \\
(1.37 \%)\end{array}$ & $14(2.5 \%)$ & $7(1.56 \%)$ & 69 (3.48\%) & $4(0.83 \%)$ & 27 (1.64\%) & $6(1.26 \%)$ & $18(1.15 \%)$ & $\begin{array}{c}228 \\
(1.96 \%)\end{array}$ & 4.21 & 0.04 \\
\hline
\end{tabular}

Note: $\mathrm{X}^{2}=$ Chi-square, $\mathrm{n}=$ number of respondents, ${ }^{*} \mathrm{p}$ value $<0.05=$ statistically significant.

ISSN: 2454-325X

DOI: https://doi.org/10.24321/2454.325X.202102 
Table 7.Distribution of Participants according to Mizaj in different Adopted Spots

\begin{tabular}{|c|c|c|c|c|c|c|c|c|c|c|c|c|c|}
\hline \multirow{2}{*}{ Variable } & \multicolumn{10}{|c|}{ Name of the Spots } & \multirow{2}{*}{$\begin{array}{l}\text { Total } \\
\text { n (\%) }\end{array}$} & \multirow{3}{*}{$\mathbf{X 2}$} & \multirow{3}{*}{ p-value } \\
\hline & Alampur & Alawalpur & Barni & Dhobghatti & Diawan & Gaurichak & Keora & Lakhna & P. Bigha & Phetiya & & & \\
\hline Mizaj & $n=1586$ & $n=538$ & $n=2334$ & $n=559$ & $n=449$ & $n=1981$ & $n=482$ & $n=1648$ & $n=478$ & $n=1566$ & $n=11621$ & & \\
\hline Damvi & $\begin{array}{c}360 \\
(22.7 \%) \\
\end{array}$ & $36(6.69 \%)$ & $\begin{array}{c}328 \\
(14.05 \%) \\
\end{array}$ & 24 (4.29\%) & $\begin{array}{c}53 \\
(11.8 \%) \\
\end{array}$ & $\begin{array}{c}275 \\
(13.88 \%) \\
\end{array}$ & $\begin{array}{c}12 \\
(2.49 \%) \\
\end{array}$ & $\begin{array}{c}255 \\
(15.47 \%) \\
\end{array}$ & $\begin{array}{c}39 \\
(8.16 \%) \\
\end{array}$ & $\begin{array}{c}227 \\
(14.5 \%) \\
\end{array}$ & $\begin{array}{c}1609 \\
(13.85 \%) \\
\end{array}$ & 24.2 & $0.001^{*}$ \\
\hline Balghami & $\begin{array}{c}953 \\
(60.09 \%) \\
\end{array}$ & $\begin{array}{c}233 \\
(43.31 \%) \\
\end{array}$ & $\begin{array}{c}1440 \\
(61.7 \%) \\
\end{array}$ & $124(22.18 \%)$ & $\begin{array}{c}393 \\
(87.53 \%)\end{array}$ & $\begin{array}{c}1246 \\
(62.9 \%)\end{array}$ & $\begin{array}{c}430 \\
(89.21 \%) \\
\end{array}$ & $\begin{array}{c}1035 \\
(62.8 \%)\end{array}$ & $\begin{array}{c}439 \\
(91.84 \%)\end{array}$ & $\begin{array}{c}959 \\
(61.24 \%) \\
\end{array}$ & $\begin{array}{c}7252 \\
(62.4 \%) \\
\end{array}$ & 87.68 & $0.001 *$ \\
\hline Safravi & $\begin{array}{c}148 \\
(9.33 \%)\end{array}$ & $\begin{array}{c}128 \\
(23.79 \%)\end{array}$ & $\begin{array}{c}245 \\
(10.5 \%)\end{array}$ & $162(28.98 \%)$ & $1(0.22 \%)$ & $\begin{array}{c}240 \\
(12.12 \%)\end{array}$ & $\begin{array}{c}20 \\
(4.15 \%)\end{array}$ & $\begin{array}{c}196 \\
(11.89 \%)\end{array}$ & $0(0 \%)$ & $\begin{array}{c}278 \\
(17.75 \%)\end{array}$ & $\begin{array}{c}1418 \\
(12.2 \%)\end{array}$ & 70.73 & $0.001 *$ \\
\hline Saudawi & $\begin{array}{c}125 \\
(7.88 \%)\end{array}$ & $\begin{array}{c}141 \\
(26.21 \%)\end{array}$ & $\begin{array}{c}321 \\
(13.75 \%)\end{array}$ & 249 (44.54\%) & $2(0.45 \%)$ & $\begin{array}{c}220 \\
(11.11 \%)\end{array}$ & $\begin{array}{c}20 \\
(4.15 \%)\end{array}$ & $\begin{array}{c}162 \\
(9.83 \%)\end{array}$ & $0(0 \%)$ & $\begin{array}{c}102 \\
(6.51 \%)\end{array}$ & $\begin{array}{c}1342 \\
(11.55 \%)\end{array}$ & 118.69 & $0.001 *$ \\
\hline
\end{tabular}

Note: $\mathrm{X}^{2}=$ Chi-square, $\mathrm{n}=$ number of respondents, ${ }^{*} \mathrm{p}$ value $<0.05=$ statistically significant.

Table 8.Distribution of Participants according to Diet in different Adopted Spots

\begin{tabular}{|c|c|c|c|c|c|c|c|c|c|c|c|c|c|}
\hline \multirow{2}{*}{ Variable } & \multicolumn{10}{|c|}{ Name of the Spots } & \multirow{2}{*}{ Total } & \multirow{3}{*}{$\mathbf{X} 2$} & \multirow{3}{*}{ p-value } \\
\hline & Alampur & Alawalpur & Barni & Dhobghatti & Diawan & Gaurichak & Keora & Lakhna & P. Bigha & Phetiya & & & \\
\hline Diet & $n=1586$ & $n=538$ & $n=2334$ & $n=559$ & $n=449$ & $n=1981$ & $n=482$ & $n=1648$ & $n=478$ & $n=1566$ & $\mathrm{~N}=11621$ & & \\
\hline Vegetarian & $\begin{array}{c}275 \\
(17.34 \%)\end{array}$ & $20(3.72 \%)$ & $\begin{array}{c}252 \\
(10.8 \%)\end{array}$ & $7(1.25 \%)$ & $\begin{array}{c}18 \\
(4.01 \%) \\
\end{array}$ & $\begin{array}{c}182 \\
(9.19 \%)\end{array}$ & $\begin{array}{c}1 \\
(0.21 \%)\end{array}$ & $\begin{array}{c}113 \\
(6.86 \%) \\
\end{array}$ & $1(0.21 \%)$ & $\begin{array}{c}170 \\
(10.86 \%)\end{array}$ & $\begin{array}{c}1039 \\
(8.94 \%)\end{array}$ & 18.9 & $0.001 *$ \\
\hline Non-Vegetarian & $\begin{array}{c}1261 \\
(79.51 \%)\end{array}$ & $\begin{array}{c}516 \\
(95.91 \%)\end{array}$ & $\begin{array}{c}2070 \\
(88.69 \%)\end{array}$ & $\begin{array}{c}552 \\
(98.75 \%)\end{array}$ & $\begin{array}{c}431 \\
(95.99 \%)\end{array}$ & $\begin{array}{c}1797 \\
(90.71 \%)\end{array}$ & $\begin{array}{c}481 \\
(99.79 \%)\end{array}$ & $\begin{array}{c}1534 \\
(93.08 \%)\end{array}$ & $\begin{array}{c}477 \\
(99.79 \%)\end{array}$ & $\begin{array}{c}1365 \\
(87.16 \%)\end{array}$ & $\begin{array}{c}10484 \\
(90.22 \%)\end{array}$ & 20.73 & $0.001 *$ \\
\hline Vegetarian + Egg & $50(3.15 \%)$ & $2(0.37 \%)$ & $12(0.51 \%)$ & $0(0 \%)$ & $0(0 \%)$ & $2(0.1 \%)$ & $0(0 \%)$ & $1(0.06 \%)$ & $0(0 \%)$ & $31(1.98 \%)$ & $98(0.84 \%)$ & 1.5 & 0.22 \\
\hline
\end{tabular}

Note: $\mathrm{X}^{2}=$ Chi-square, $\mathrm{n}=$ number of respondents, ${ }^{*} \mathrm{p}$ value $<0.05=$ statistically significant.

Table 9.Distribution of Participants according to Occupation in different Adopted Spots

\begin{tabular}{|c|c|c|c|c|c|c|c|c|c|c|c|c|c|}
\hline \multirow{2}{*}{ Variable } & \multicolumn{10}{|c|}{ Name of the Spots } & Total & \multirow{3}{*}{$X 2$} & \multirow{3}{*}{$p$-value } \\
\hline & Alampur & Alawalpur & Barni & Dhobghatti & Diawan & Gaurichak & Keora & Lakhna & P. Bigha & Phetiya & & & \\
\hline Occupation & $n=1586$ & $n=538$ & $n=2334$ & $n=559$ & $n=449$ & $n=1981$ & $n=482$ & $n=1648$ & $n=478$ & $n=1566$ & $\mathrm{~N}=11621$ & & \\
\hline None & $\begin{array}{c}164 \\
(10.34 \%)\end{array}$ & 53 (9.85\%) & $\begin{array}{c}315 \\
(13.5 \%)\end{array}$ & $36(6.44 \%)$ & $\begin{array}{c}30 \\
(6.68 \%)\end{array}$ & $\begin{array}{c}195 \\
(9.84 \%)\end{array}$ & $\begin{array}{c}41 \\
(8.51 \%)\end{array}$ & $\begin{array}{c}165 \\
(10.01 \%)\end{array}$ & $\begin{array}{c}44 \\
(9.21 \%)\end{array}$ & $\begin{array}{c}202 \\
(12.9 \%)\end{array}$ & $\begin{array}{c}1245 \\
(10.71 \%)\end{array}$ & 0.44 & 0.51 \\
\hline
\end{tabular}

ISSN: 2454-325X DOI: https://doi.org/10.24321/2454.325X.202102 
Ahmad F et al.

Int. J. Preven. Curat. Comm. Med. 2021; 7(1)

\begin{tabular}{|c|c|c|c|c|c|c|c|c|c|c|c|c|c|}
\hline Landholder & $5(0.32 \%)$ & $2(0.37 \%)$ & $22(0.94 \%)$ & $1(0.18 \%)$ & $0(0 \%)$ & $0(0 \%)$ & $0(0 \%)$ & $10(0.61 \%)$ & $0(0 \%)$ & $6(0.38 \%)$ & $46(0.4 \%)$ & 0.01 & 0.92 \\
\hline $\begin{array}{l}\text { Agricultural } \\
\text { Labourer }\end{array}$ & $\begin{array}{c}299 \\
(18.85 \%)\end{array}$ & $\begin{array}{c}106 \\
(19.7 \%)\end{array}$ & $\begin{array}{c}250 \\
(10.71 \%)\end{array}$ & $\begin{array}{c}116 \\
(20.75 \%)\end{array}$ & $\begin{array}{c}81 \\
(18.04 \%)\end{array}$ & $\begin{array}{c}291 \\
(14.69 \%)\end{array}$ & $\begin{array}{c}112 \\
(23.24 \%)\end{array}$ & $\begin{array}{c}285 \\
(17.29 \%)\end{array}$ & $\begin{array}{c}105 \\
(21.97 \%)\end{array}$ & $\begin{array}{c}343 \\
(21.9 \%)\end{array}$ & $\begin{array}{c}1988 \\
(17.11 \%)\end{array}$ & 2.68 & 0.1 \\
\hline $\begin{array}{l}\text { Unskilled } \\
\text { Labourer }\end{array}$ & $\begin{array}{c}118 \\
(7.44 \%)\end{array}$ & 8 (1.49\%) & $\begin{array}{c}173 \\
(7.41 \%)\end{array}$ & $41(7.33 \%)$ & $\begin{array}{c}20 \\
(4.45 \%)\end{array}$ & $111(5.6 \%)$ & $\begin{array}{c}22 \\
(4.56 \%)\end{array}$ & $\begin{array}{c}170 \\
(10.32 \%)\end{array}$ & $\begin{array}{c}12 \\
(2.51 \%)\end{array}$ & 96 (6.13\%) & 771 (6.63\%) & 24.13 & $0.001 *$ \\
\hline $\begin{array}{c}\text { Skilled } \\
\text { Labourer }\end{array}$ & 19 (1.2\%) & $16(2.97 \%)$ & $18(0.77 \%)$ & 9 (1.61\%) & 5 (1.11\%) & 39 (1.97\%) & $2(0.41 \%)$ & $34(2.06 \%)$ & $\begin{array}{c}2 \\
(0.42 \%)\end{array}$ & 25 (1.6\%) & 169 (1.45\%) & 9.09 & $0.001 *$ \\
\hline Business & $32(2.02 \%)$ & 9 (1.67\%) & $50(2.14 \%)$ & $4(0.72 \%)$ & $6(1.34 \%)$ & 37 (1.87\%) & $1(0.21 \%)$ & 27 (1.64\%) & $0(0 \%)$ & 38 (2.43\%) & 204 (1.76\%) & 0.02 & 0.89 \\
\hline Student & $\begin{array}{c}357 \\
(22.51 \%)\end{array}$ & $\begin{array}{c}55 \\
(10.22 \%)\end{array}$ & $\begin{array}{c}256 \\
(10.97 \%)\end{array}$ & $56(10.02 \%)$ & $\begin{array}{c}54 \\
(12.03 \%)\end{array}$ & $\begin{array}{c}262 \\
(13.23 \%)\end{array}$ & $\begin{array}{c}15 \\
(3.11 \%)\end{array}$ & $\begin{array}{c}212 \\
(12.86 \%)\end{array}$ & $\begin{array}{c}47 \\
(9.83 \%)\end{array}$ & $\begin{array}{c}264 \\
(16.86 \%)\end{array}$ & $\begin{array}{c}1578 \\
(13.58 \%)\end{array}$ & 5.41 & 0.02 \\
\hline Housewife & $\begin{array}{c}566 \\
(35.69 \%)\end{array}$ & $\begin{array}{c}280 \\
(52.04 \%)\end{array}$ & $\begin{array}{c}1228 \\
(52.61 \%)\end{array}$ & $\begin{array}{c}292 \\
(52.24 \%)\end{array}$ & $\begin{array}{c}253 \\
(56.35 \%)\end{array}$ & $\begin{array}{c}1024 \\
(51.69 \%)\end{array}$ & $\begin{array}{c}284 \\
(58.92 \%)\end{array}$ & $\begin{array}{c}719 \\
(43.63 \%)\end{array}$ & $\begin{array}{c}266 \\
(55.65 \%)\end{array}$ & $\begin{array}{c}574 \\
(36.65 \%)\end{array}$ & $\begin{array}{c}5486 \\
(47.21 \%)\end{array}$ & 5.3 & 0.020 \\
\hline Unemployed & $1(0.06 \%)$ & $0(0 \%)$ & $2(0.09 \%)$ & $0(0 \%)$ & $0(0 \%)$ & $2(0.1 \%)$ & $1(0.21 \%)$ & $0(0 \%)$ & $0(0 \%)$ & $0(0 \%)$ & $6(0.05 \%)$ & 0.29 & 0.59 \\
\hline None & $\begin{array}{c}164 \\
(10.34 \%)\end{array}$ & $53(9.85 \%)$ & $\begin{array}{c}315 \\
(13.5 \%)\end{array}$ & $36(6.44 \%)$ & $\begin{array}{c}30 \\
(6.68 \%)\end{array}$ & $\begin{array}{c}195 \\
(9.84 \%)\end{array}$ & $\begin{array}{c}41 \\
\text { (8.51\%) }\end{array}$ & $\begin{array}{c}165 \\
(10.01 \%)\end{array}$ & $\begin{array}{c}44 \\
(9.21 \%)\end{array}$ & $\begin{array}{c}202 \\
(12.9 \%)\end{array}$ & $\begin{array}{c}1245 \\
(10.71 \%)\end{array}$ & 0.44 & 0.51 \\
\hline
\end{tabular}

Note: $\mathrm{X}^{2}=$ Chi-square, $\mathrm{n}=$ number of respondents, ${ }^{*} \mathrm{p}$ value $<0.05=$ statistically significant.

Table I O.Distribution of Participants according to Addiction in different Adopted Spots

\begin{tabular}{|c|c|c|c|c|c|c|c|c|c|c|c|c|c|}
\hline \multirow{3}{*}{$\begin{array}{l}\text { Variable } \\
\text { Addiction }\end{array}$} & \multicolumn{10}{|c|}{ Name of the Spots } & \multirow[t]{2}{*}{ Total } & \multirow{3}{*}{$x 2$} & \multirow{3}{*}{ p-value } \\
\hline & \multirow{2}{*}{$\begin{array}{c}\text { Alampur } \\
\mathrm{n}=1586\end{array}$} & \multirow{2}{*}{$\begin{array}{c}\text { Alawalpur } \\
n=538\end{array}$} & \multirow{2}{*}{$\begin{array}{c}\text { Barni } \\
\mathrm{n}=\mathbf{2 3 3 4}\end{array}$} & \multirow{2}{*}{$\begin{array}{c}\text { Dhobghatti } \\
n=559\end{array}$} & \multirow{2}{*}{$\begin{array}{c}\text { Diawan } \\
n=449\end{array}$} & \multirow{2}{*}{$\begin{array}{c}\text { Gaurichak } \\
\text { n=1981 }\end{array}$} & \multirow{2}{*}{$\begin{array}{l}\text { Keora } \\
n=482\end{array}$} & \multirow{2}{*}{$\begin{array}{l}\text { Lakhna } \\
n=1648\end{array}$} & \multirow{2}{*}{$\begin{array}{c}\text { P. Bigha } \\
n=478\end{array}$} & \multirow{2}{*}{$\begin{array}{l}\text { Phetiya } \\
n=1566\end{array}$} & & & \\
\hline & & & & & & & & & & & $N=11621$ & & \\
\hline None & $\begin{array}{c}1060 \\
(66.83 \%)\end{array}$ & $\begin{array}{c}362 \\
(67.29 \%)\end{array}$ & $\begin{array}{c}1817 \\
(77.85 \%)\end{array}$ & $\begin{array}{c}377 \\
(67.44 \%)\end{array}$ & $\begin{array}{c}313 \\
(69.71 \%)\end{array}$ & $\begin{array}{c}1472 \\
(74.31 \%)\end{array}$ & $\begin{array}{c}310 \\
(64.32 \%)\end{array}$ & $\begin{array}{c}1106 \\
(67.11 \%)\end{array}$ & $\begin{array}{c}321 \\
(67.15 \%)\end{array}$ & $\begin{array}{c}948 \\
(60.54 \%)\end{array}$ & $\begin{array}{c}8086 \\
(69.58 \%)\end{array}$ & 1.4 & 0.24 \\
\hline Tobacco & $\begin{array}{c}504 \\
(31.78 \%)\end{array}$ & $\begin{array}{c}176 \\
(32.71 \%)\end{array}$ & $\begin{array}{c}499 \\
(21.38 \%)\end{array}$ & $\begin{array}{c}181 \\
(32.38 \%)\end{array}$ & $\begin{array}{c}136 \\
(30.29 \%)\end{array}$ & $\begin{array}{c}497 \\
(25.09 \%)\end{array}$ & $\begin{array}{c}172 \\
(35.68 \%)\end{array}$ & $\begin{array}{c}531 \\
(32.22 \%)\end{array}$ & $\begin{array}{c}157 \\
(32.85 \%)\end{array}$ & $\begin{array}{c}598 \\
(38.19 \%)\end{array}$ & $\begin{array}{c}3451 \\
(29.7 \%)\end{array}$ & 2.46 & 0.12 \\
\hline Snuff & $11(0.69 \%)$ & $0(0 \%)$ & $11(0.47 \%)$ & $0(0 \%)$ & $0(0 \%)$ & $6(0.3 \%)$ & $0(0 \%)$ & $4(0.24 \%)$ & $0(0 \%)$ & $8(0.51 \%)$ & $40(0.34 \%)$ & 1.95 & 0.16 \\
\hline Smoking & $10(0.63 \%)$ & $0(0 \%)$ & $7(0.3 \%)$ & $1(0.18 \%)$ & $0(0 \%)$ & $5(0.25 \%)$ & $0(0 \%)$ & $6(0.36 \%)$ & $0(0 \%)$ & $10(0.64 \%)$ & $39(0.34 \%)$ & 1.9 & 0.17 \\
\hline Bhang & $1(0.06 \%)$ & $0(0 \%)$ & $0(0 \%)$ & $0(0 \%)$ & $0(0 \%)$ & $0(0 \%)$ & $0(0 \%)$ & $0(0 \%)$ & $0(0 \%)$ & $0(0 \%)$ & $1(0.01 \%)$ & 0.05 & 0.82 \\
\hline Alcohol & $0(0 \%)$ & $0(0 \%)$ & $0(0 \%)$ & $0(0 \%)$ & $0(0 \%)$ & 1 (0.05\%) & $0(0 \%)$ & $1(0.06 \%)$ & $0(0 \%)$ & $2(0.13 \%)$ & $4(0.03 \%)$ & 0.19 & 0.66 \\
\hline
\end{tabular}

Note: $X^{2}=$ Chi-square, $n=$ number of respondents, ${ }^{*} p$ value $<0.05=$ statistically significant.

ISSN: 2454-325X

DOI: https://doi.org/10.24321/2454.325X.202102 


\section{Conclusion}

It can be concluded that majority of SCS is living in rural areas with unique physical, socio-economic and cultural environment. There is significant difference in the age group of SC population. While there were no significant difference with respect to gender, others religion and addiction behaviour was noticed. Preferred food habit was mixed mostly. The main occupation of the SC people is farming. They have also adopted other available occupations for improving their status. Most of the SC population which was under observation, belong to balghami temperament. SC population are aware of harmful effect of addiction and majority of them are non-addictive.

\section{Acknowledgement}

The authors are indebted to the Director General, Central Council for Research in Unani Medicine, New Delhi for sponsoring the study. Authors are also thankful to all the local Mukhiyas, Pramukhs and Scheduled Caste people for their support as well as entire staff of SC-SP programme at RRIUM, Patna.

\section{Conflict of Interest: None}

\section{References}

1. Census of India, [https://censusindia.gov.in/census_ and_you/scheduled_castes_and_sceduled_tribes. aspx]. Office of the Registrar General \& Census Commissioner, Delhi. Ministry of Home Affairs, Government of India. 2011. [cited 2020 Oct 26, Nov 12].

2. Scheduled Castes Population - Demography of Scheduled Castes. [http://censusmp.nic.in/censusmp/ Data/PCA_DATA/007\%20-\%20Chapter\%20-\%205\%20 -\%20SC\%20.pdf ]. Analytical Report on Primary Census Abstract. [cited 2020 Oct 26].

3. Department of Personnel \& Training [https://dopt.gov. in/sites/default/files/ch-11.pdf]. Special representation in Services of SC/ST. [cited 2020 Oct 26, Nov 12].

4. Article 341 in the Constitution of India 1949 Central Government Act [https://indiankanoon.org/ doc/68762/]. [cited 2020 Oct 26, Nov 12].

5. Chouhan P. A study on literacy and educational attainment of scheduled castes population in Maldah District of West Bengal, India. J Geo Reg Plann. 2013; 6(1): 19-30. [https://doi.org/10.5897/JGRP12.028]. [cited 2020 Oct 26, Nov 12].

6. Ministry of Social Justice and Empowerment [http:// socialjustice.nic.in/writereaddata/UploadFile/ HANDBOOKSocialWelfareStatistice2018.pdf]. Handbook on Social Welfare Statistics. Government of India - Ministry of Social Justice \& Empowerment Department of Social Justice and Empowerment Plan Division. New Delhi. 2018. [cited 2020 Oct 26, Nov 12].

7. Patna - About District [https://patna.nic.in/about- district/]. [cited 2020 Nov 16].

8. Census India [https://www.censusindia.co.in/district/ vaishali-district-bihar-220]. Vaishali District Population, Caste, Religion Data (Bihar) - Census 2011. [cited 2020 Nov 16].

9. Census India [https://www.censusindia.co.in/districts]. Nalanda District [Internet]. [cited 2020 Nov 16].

10. Central Council for Research in Unani Medicine [https:// ccrum.res.in/UserView/index?mid=1745]. Operational guidelines, Mobile Health Care Programme under Scheduled Caste-Sub plan (SC-SP). Ministry of AYUSH, Government of India, 2017. [cited 2020 Nov 01]. 